\title{
Demonstration of protein-fragment complementation assay using purified firefly luciferase fragments
}

Yuki Ohmuro-Matsuyama ${ }^{1,2,3^{\dagger}}$, Chan-I Chung ${ }^{1 \dagger}$ and Hiroshi Ueda ${ }^{1,3^{*}}$

\begin{abstract}
Background: Human interactome is predicted to contain 150,000 to 300,000 protein-protein interactions, (PPIs). Protein-fragment complementation assay (PCA) is one of the most widely used methods to detect PPI, as well as Förster resonance energy transfer (FRET). To date, successful applications of firefly luciferase (Fluc)-based PCA have been reported in vivo, in cultured cells and in cell-free lysate, owing to its high sensitivity, high signal-to -background (S/B) ratio, and reversible response. Here we show the assay also works with purified proteins with unexpectedly rapid kinetics.

Results: Split Fluc fragments both fused with a rapamycin-dependently interacting protein pair were made and expressed in E. coli system, and purified to homogeneity. When the proteins were used for PCA to detect rapamycin-dependent PPI, they enabled a rapid detection ( 1 s) of PPI with high S/B ratio. When Fn7-8 domains (7 nm in length) that was shown to abrogate GFP mutant-based FRET was inserted between split Fluc and FKBP12 as a rigid linker, it still showed some response, suggesting less limitation in interacting partner's size. Finally, the stability of the probe was investigated. Preincubation of the probes at 37 degreeC up to $1 \mathrm{~h}$ showed marked decrease of the luminescent signal to $1.5 \%$, showing the limited stability of this system.

Conclusion: Fluc PCA using purified components will enable a rapid and handy detection of PPIs with high S/B ratio, avoiding the effects of concomitant components. Although the system might not be suitable for large-scale screening due to its limited stability, it can detect an interaction over larger distance than by FRET. This would be the first demonstration of Fluc PCA in vitro, which has a distinct advantage over other PPI assays. Our system enables detection of direct PPIs without risk of perturbation by PPI mediators in the complex cellular milieu.
\end{abstract}

Keywords: Protein-protein interaction, Firefly luciferase, Bioluminescence, Protein fragment complementation assay, Thermostability, In vitro diagnostics

\section{Background}

It is estimated that there are about 150,000 to 300,000 protein-protein interactions (PPI) in human interactome $[1,2]$. In order to discover and investigate PPI, exploration of PPI assays is considered highly important. Among various PPI assays, protein-fragment complementation assay (PCA) is a simple and user-friendly

\footnotetext{
* Correspondence: ueda@res.titech.ac.jp

${ }^{\dagger}$ Equal contributors

'Department of Chemistry and Biotechnology, School of Engineering, The University of Tokyo, 7-3-1 Hongo, Bunkyo-ku, Tokyo 113-8656, Japan ${ }^{3}$ Chemical Resources Laboratory, Tokyo Institute of Technology, R1-18, 4259 Nagatsuta-cho, Midori-ku, Yokohama 226-8501, Japan

Full list of author information is available at the end of the article
}

method [3]. In PCA, a reporter protein, such as a green fluorescent protein (GFP) variant $[4,5]$ and a luciferase [6-10] is dissected to a split from, and each split reporter protein is fused to an interacting partner (Figure 1). While the split reporter proteins have low reporter activity when they exist far apart (Figure 1A), they reconstitute and recover the activity when the proteins come close due to the association of interacting partner (Figure 1B). In general, due to its low background activity, PCA displays high signal to background ratio (S/B). Especially, bioluminescence-based PCAs such as splitluciferase reporters shows reversible property [11], in contrast to the irreversible nature of the reassembled GFP-like fluorescent proteins [5]. In addition, since






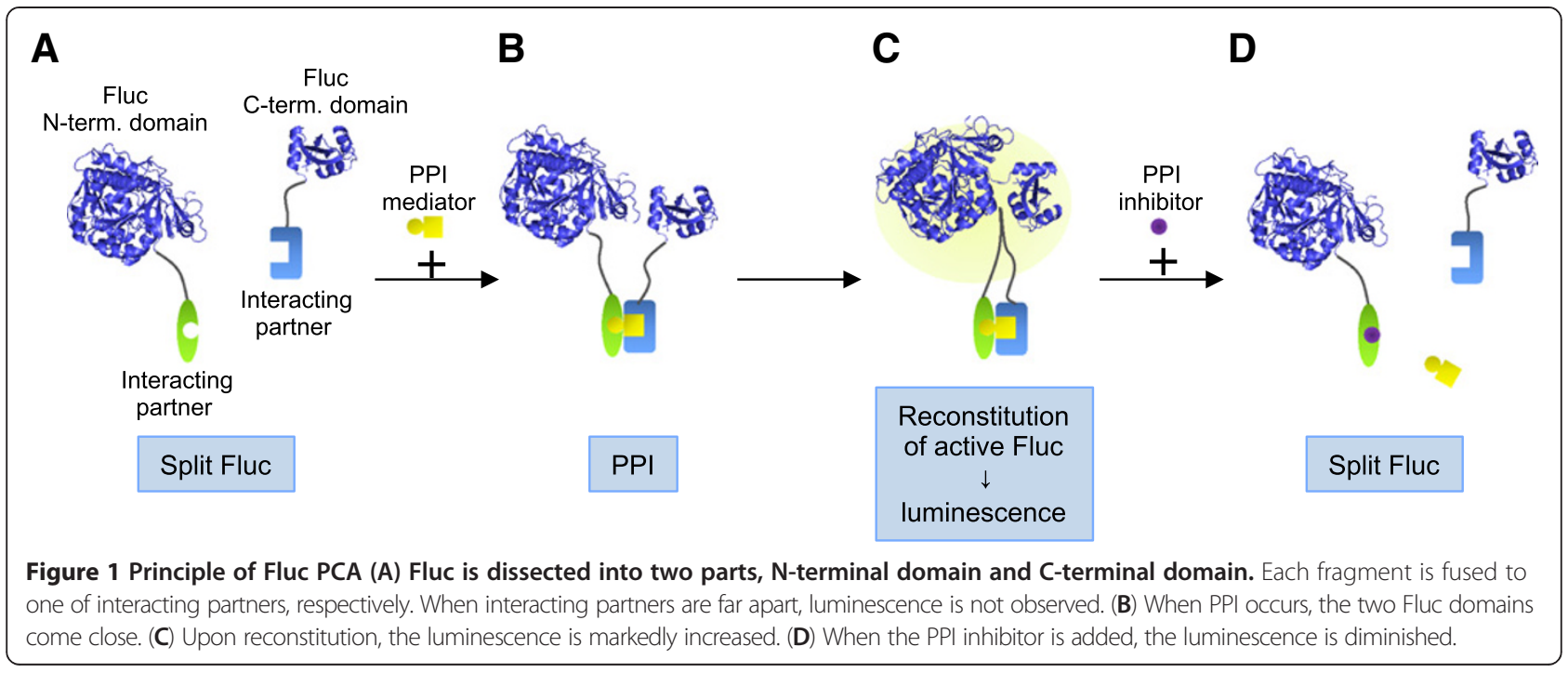

luciferases need no excitation light at shorter wavelength for their detection, the assay enables noninvasive and sensitive optical imaging in living cells and animals.

While PPI is conveniently assayed by PCA in vivo and in cultured cells, it is often desirable to be performed also in vitro, to know whether the interaction is direct or not, since many PPI mediators, inhibitors and enhancers may exist in the complex cellular milieu. To date, Porter et al. successfully utilized a cell-free transcription/translation system for performing firefly luciferase (Fluc)-based PCA in vitro [11]. Their probes can be prepared in shorter time period than the probes expressed in E. coli, yeast or mammalian cells. However, the assay needs expensive cell-free lysate, and whose components always have a risk to affect PPI. Here we report a PCA using purified interacting proteins fused with split Fluc proteins, to detect PPI in a defined solution. We also examined the stability of the probes, and the detectable distance between the interacting partners. To the best of our knowledge, this will be the first investigation of pure in vitro PPI, based on Fluc PCA.

\section{Results}

For the PPI to be investigated, we first selected a wellknown interacting domain pair of FKBP12 and FRB. FKBP12 is a $12 \mathrm{kD}$ domain of FK506 binding protein (FKBP), which is able to associate with FKBP-rapamycin -associated protein (FRB) depending on an antibiotic, rapamycin (PPI mediator in Figure 1) $[12,13]$. To this end, FKBP12 or FRB gene was fused to the $5^{\prime}$ of Fluc Nterminal (1-437 in amino acid, aa) or C-terminal domain (394-547 in aa) gene, yielding four types of fusion protein genes (FKBP/N, FKBP/C, FRB/N, and FRB/C). The genes were inserted to $\mathrm{pET} 32 \mathrm{~b}$ vector, and the thioredoxin-fused proteins were expressed in the soluble fraction of $E$. coli BL21(DE3, pLysS) and purified by an immobilized metal affinity chromatography (Figure 2A). The two interacting pairs (FKBP/N - FRB/C and FKBP/ $\mathrm{C}-\mathrm{FRB} / \mathrm{N}$ ) were mixed at $50 \mathrm{nM}$ each, and equimolar rapamycin was added to the mixtures. Just after adding the two substrates ATP and luciferin, the luminescent intensity was measured by a luminometer at $0.1 \mathrm{~s}$ intervals for $4 \mathrm{~s}$. As a result, the intensity of the interacting pairs added with rapamycin showed a marked increase. In contrast, the pairs without rapamycin displayed very low luminescence (Figure 2B). In addition, non-interacting protein pairs (FKBP/N - FKBP/C, and FRB/N - FRB/C), as well as each fusion protein alone exhibit very low luminescence even in the presence of rapamycin (Figure 2C). The results clearly showed that the PPI can be specifically detected with high S/B ratio using purified probes.

To assess the degree of spontaneous Fluc reconstitution, the assay with one of the best interacting pairs $\mathrm{FRB} / \mathrm{N}$ and $\mathrm{FKBP} / \mathrm{C}$ was performed at several probe concentrations (Figure 3). At all the concentrations tested, the luminescent signals increased rapamycin-dependently (Figure 3, A-D). The resulting maximum signal/background (S/B) ratio increased to as high as 130 when at the probe concentration increased up to $250 \mathrm{nM}$, while the ratio at 750 $\mathrm{nM}$ was slightly lower (Figure 3E). This was possibly because the high probe concentration rather promoted spontaneous Fluc reconstitution without rapamycin. When the rapamycin dose-response at $50 \mathrm{nM}$ probe concentration was examined, the limit of detection was determined as $250 \mathrm{pM}$ (Figure 3F).

For Fluc PCA, several successful split sites are reported to date. To investigate on this issue, another pair of $\mathrm{N}$-terminal domain (1-398) together with the same Cterminal domain (394-547) was employed according to a previous attempt that gave good response in vivo (Additional file 1: Figure S1) [14]. We could express and purify the new $\mathrm{N}$-domain similarly (Additional file 1 : 



Figure 2 Fluc PCA using purified probes (A) Purification of the Fluc PCA probes. Lane 1, FKBP/N; Lane 2, FRB/N; Lane 3, FKBP/C; Lane 4, FRB/C. (B) PCA using the purified probes at $50 \mathrm{nM}$ each, with/without equimolar rapamycin (Rap). Average and SD values of three samples are shown. (C) Control experiments using non-interacting partners. All the conditions are the same as above.

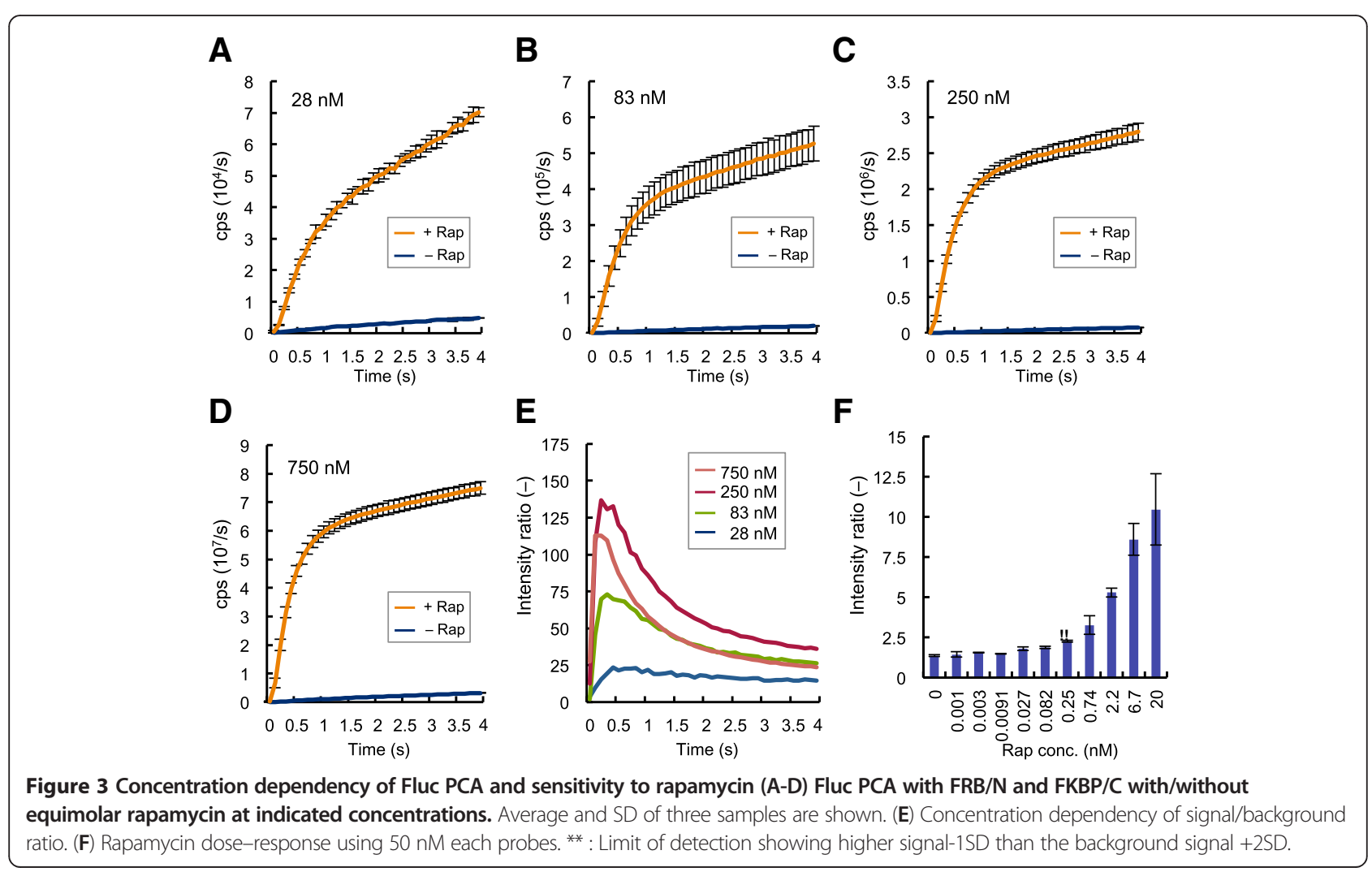


Figure S1A), and obtained clear rapamycin-dependent signals at several concentrations of $\mathrm{FRB} / \mathrm{N}(1-398)$ FKBP/C pair (Additional file 1: Figure S1, B-E). However, compared with the FRB/N(1-437) - FKBP/C(394-547) pair, the signal intensity was lower, and the resultant S/B ratio was rather unstable, albeit not low (Additional file 1: Figure S1F). This was probably because of the instability of the N-domain (1-398), which is reflected to its degradation products observed in Additional file 1: Figure S1A.

Since rapamycin-dependent FKBP-FRB interaction is one of very strong protein-protein interactions [15], another cellular interaction of Mdm2 and p53 was investigated (Figure 4). Mdm2 oncoprotein is a cellular inhibitor of the p53 tumor suppressor in that it can bind the transactivation domain of p53 and down-regulates its ability to activate transcription. In certain cancers, mdm 2 amplification is a common event and contributes to the inactivation of p53 [16]. The probes fused to the trans-activation domain of p53 and Mdm2 (p53/C, p53/N, Mdm2/C and $\mathrm{Mdm} 2 / \mathrm{N}$ ) were constructed in the same way as $\mathrm{FKBP} / \mathrm{C}$ and FRB/N (1-437) (Figure 4A). This time, both the signal intensity and $\mathrm{S} / \mathrm{B}$ ratio elevated depending on the concentration of the probes (Figure 4, B-F). Then we investigated whether the in vitro Fluc PCA could detect the reversibility of interaction. After we mixed the probes $\mathrm{p} 53 / \mathrm{C}$ and
$\mathrm{Mdm} 2 / \mathrm{N}$ at $100 \mathrm{nM}$ each, Nutlin-3, a known inhibitor of p53-Mdm2 interaction, was added and measured for the Fluc activity (Figure 4G). The result clearly showed a Nutlin-3 concentration dependent luminescence inhibition with calculated $\mathrm{IC}_{50}$ of $368 \pm 25 \mathrm{nM}$. Although the value is higher than the reported one (90 nM) [17], this might be due to the racemization of Nutlin-3 used. Taken together, these results clearly showed the generality of the assay to detect PPIs including transient interactions.

Due to the nature of split enzyme, we reasoned that the instability of the purified probes could be a possible demerit of Fluc-based PCA. To examine the stability of the probes, the mixtures of $100 \mathrm{nM} \mathrm{FKBP} / \mathrm{C}$ and 100 $\mathrm{nM} \mathrm{FRB} / \mathrm{N}$ with or without equimolar rapamycin were incubated at $37^{\circ} \mathrm{C}$ for $0-60 \mathrm{~min}$, and applied to the luminescence assay (Additional file 2: Figure S2). As a result, the incubation resulted in a time-dependent decrease in luminescent intensity of the pair added with rapamycin (Additional file 2: Figure S2A). As short as $15 \mathrm{~min}$ incubation resulted in the activity loss to less than one-third of that before incubation. The longer incubation for 60 min resulted in virtually no signal difference between the pairs with and without rapamycin (Additional file 2: Figure S2, B and C). These results clearly indicate that the Fluc PCA probes have a limited stability in this

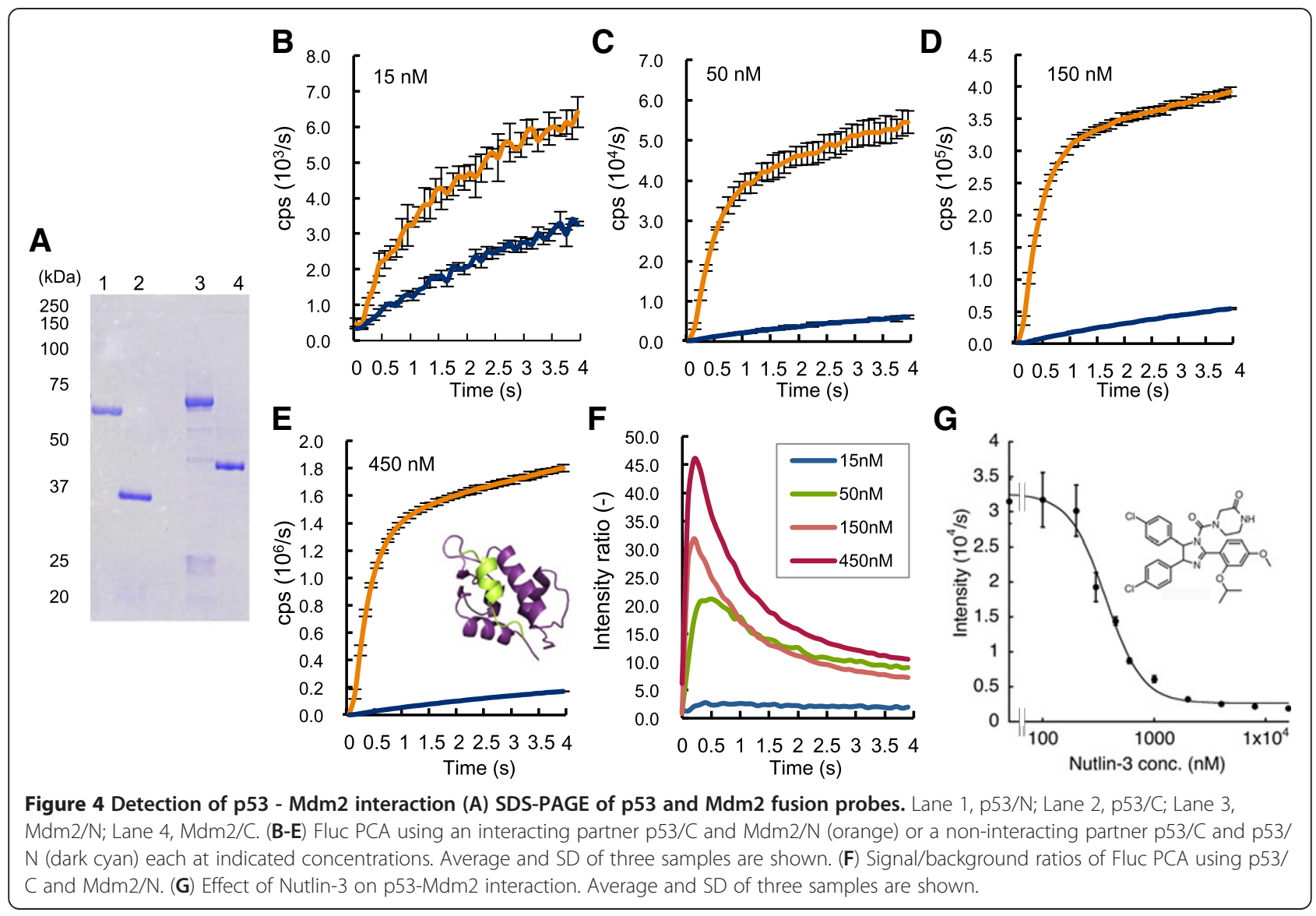


purified milieu. However, the results might also reflect their limited stability in vivo or in lysate, which is probably sequestered by the newly synthesized probes.

Förster resonance energy transfer (FRET) is an excellent PPI detection method both in vitro and in vivo, and regarded as a standard tool in this area [18]. However, FRET has a fundamental limitation that it only occurs over rather short distance of several $\mathrm{nm}$ between the donor and the acceptor fluorophores, depending on Förster distance $R_{0}$, which is determined a priori by the dye combination used $[19,20]$. Therefore, the interaction between proteins with large dimensions is a challenging task for FRET, due to the possible long distance between the attached dyes including fluorescent proteins. To compare Fluc PCA with FRET, we examined the effect of distance between interacting partners. Previously, we introduced several helix-forming peptide linkers with various lengths between EGFP and EBFP, and those linkers showed the ability to control the distance between interacting partners [21]. According to this, a similar helix-forming linker, 4repeats of Asp-Asp-Ala-Lys-Lys $(4 \times$ DDAKK), was inserted between FKBP12 and Fluc C-terminal domain. The SDS polyacrylamide gel electrophoresis of the fusion proteins (Figure 5A) suggests effective separation of FKBP12 and Fluc C-terminal domain by the inserted linkers. Using 100 $\mathrm{nM}$ each mixture of $\mathrm{FKBP} / 4 \times \mathrm{DDAKK} / \mathrm{C}, \mathrm{FRB} / \mathrm{N}$ and rapamycin, PPI was detected with a high $\mathrm{S} / \mathrm{B}$ ratio with a slight reduction compared with the pairs without helical linkers (Figure 5B). In addition, when a linker with 7 repeats of Asp-Asp-Ala-Lys-Lys $(7 \times$ DDAKK) was inserted between FKBP12 and Fluc C-terminal domain, high S/B ratio was still maintained (Figure $5, \mathrm{~A}$ and $\mathrm{C}$ ).

For a further examination, the 7th and 8th domains of fibronectin type III (Fn7-8) with a $7 \mathrm{~nm} \mathrm{N-C} \mathrm{terminal}$ distance was inserted between FKBP12 and Fluc Cterminal domain, as a rigid linker. From the SDS-PAGE of purified proteins, while the mobility of the reporter was lowered by the insertion of Fn7-8, no significant reduction in the yield and purity was observed (Figure 6A, Lanes 1 and 4). Ohashi et al. reported that the insertion of Fn7-8 domains between the fluorescent protein pair CyPet and Ypet results in barely detectable FRET signal. [22]. To confirm this, Fn7-8 was inserted between FKBP12 and a brighter CFP variant Cerulean (FKBP/Fn78/Cerulean) (Figure 6A, Lanes 2 and 3). As expected, very weak FRET signal derived of FKBP/Fn7-8/Cerulean and FRB-fused Ypet (FRB/Ypet) was observed, although a distinct FRET-derived peak was observed for the construct without Fn7-8 insertion (Figure 6B).

On the other hand, the PCA pair of FKBP/Fn7-8/C and $F R B / N$ at $750 \mathrm{nM}$ displayed a moderate but significant S/B ratio, clearly showing its superiority over FRET, although the observed rapamycin dependency was not so clear when reduced amounts $(100 \mathrm{nM})$ of the probes were used (Figure 6C). Nevertheless, the Fluc PCA system was shown to work for detecting PPI over longer distance, which was not detectable by FRET.

\section{Discussion}

Previously, Fluc-based PCA has been utilized as a sensitive tool to investigate PPI in vivo and in cultured cells. However, considering possible disturbance by the coexisting components acting as PPI mediators, enhancers and inhibitors, PPI assay in vitro will also give us invaluable information. Furthermore, the in vitro assay has the possibility to study PPIs between pathogen-derived cytotoxic proteins and host proteins, which are normally impossible to perform in vivo. In this study, we showed that Fluc PCA also works with purified elements. The system displayed high $\mathrm{S} / \mathrm{B}$ ratio and unexpectedly rapid response $(\sim 1 \mathrm{~s})$. In $\mathrm{PCA}$, the background signal due to nonspecific activation of the reporter can be a problem. As

\section{A}



B

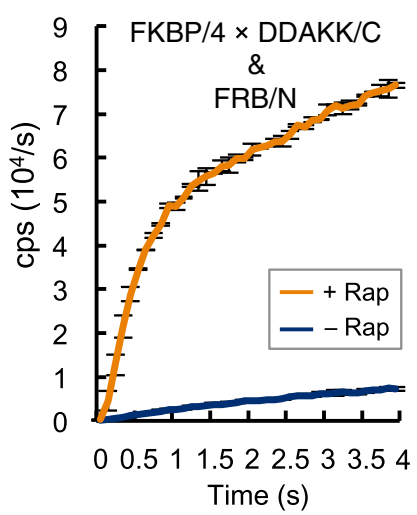

C

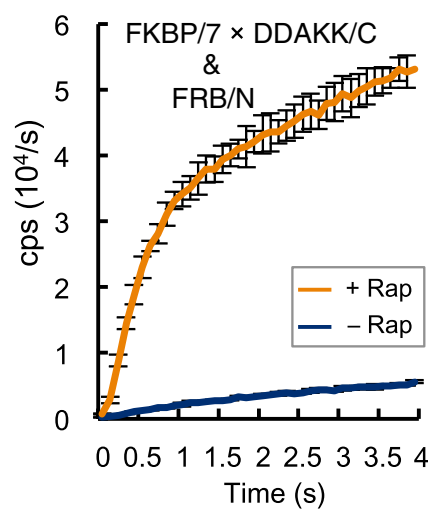

Figure 5 Insertion of helix linkers (A) SDS-PAGE of the probes inserted with helix linkers. Lane 1, FKBP/C; Lane 2, FKBP / $4 \times$ DDAKK /C; Lane 3, FKBP/ $7 \times$ DDAKK /C. (B) PCA using the probe with $4 \times$ DDAKK. The probes (100 nM each) with/without equimolar rapamycin was used $(n=3)$. (C) PCA using the probe with $7 \times$ DDAKK. The condition is the same as in $(\mathbf{B})$. 


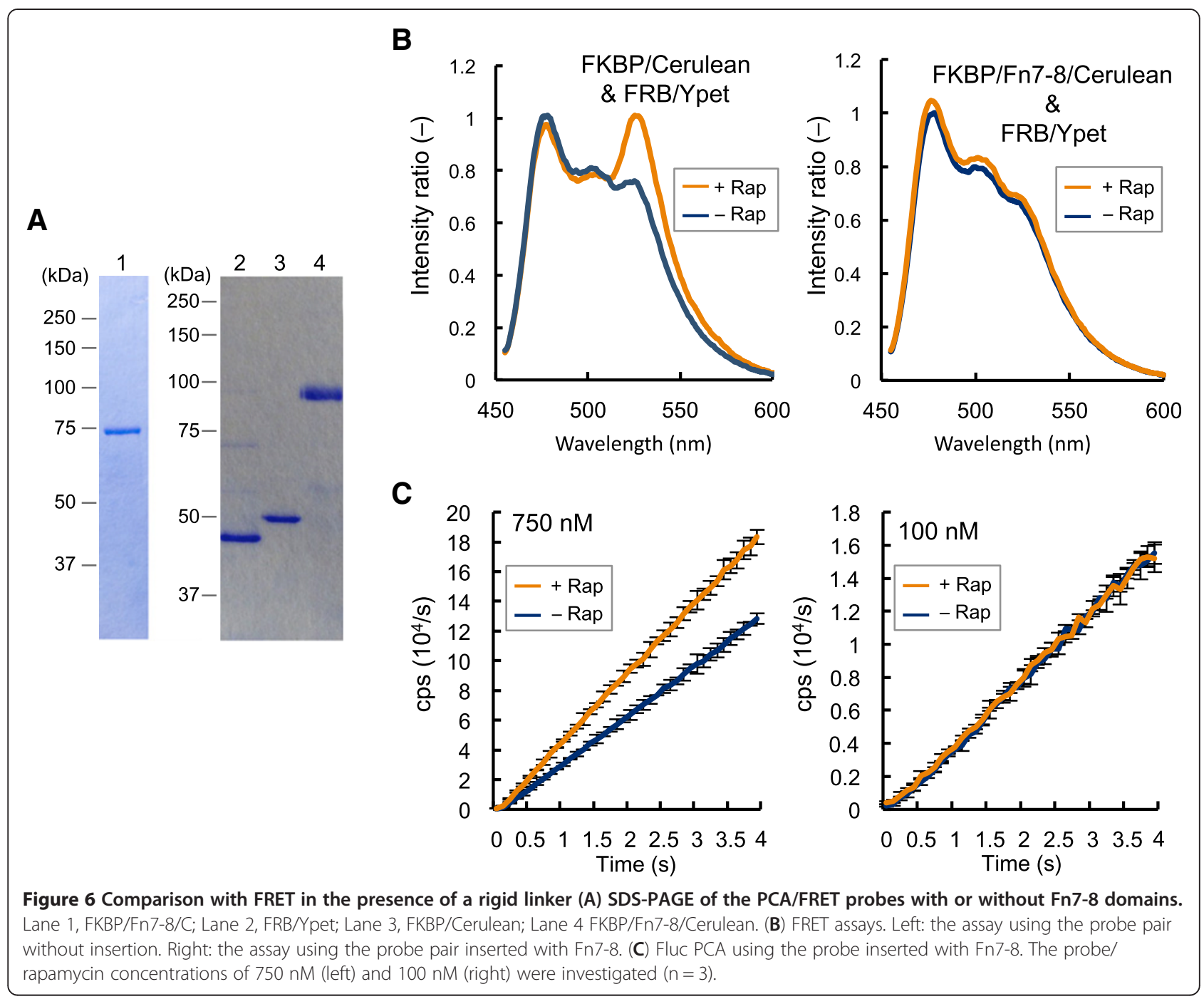

in Figure 2C, luminescent intensities of non-interacting pairs were slightly higher than those of single probe alone, probably reflecting the nonspecific reconstitution of split Fluc. However, when the specific PPI was induced by rapamycin, it resulted in markedly higher signal, giving high S/B ratio. Therefore, in practice the nonspecific signals hardly interfere with the PPI detection (Figure 2).

The only but not easily avoidable drawback of this system is the instability of probe as shown in Additional file 2: Figure S2. Apparently, the system is not very suitable for large-scale screening that needs longer sample preparation time. However, proper cooling of the reagents and samples during the preparation period will surely reduce such inactivation. Therefore, we think that the system has a potential for low-cost and highthroughput drug screenings. On the other hand, a distinct merit of this system is robustness to the interaction detection over longer distance. In fact, insertion of either long helical linker or a rigid large protein domain did not deteriorate PPI detection, while FRET signal was barely detectable when Fn7-8 was inserted (Figure 6B). These results show a lesser limitation in distance between interacting partners in PCA than in FRET. Therefore, this system will be available for the interactions between large proteins that cannot be assayed by FRET. While the reason for observed nonspecific PPI signal remains unclear, it may be due to the interaction between the inserted sequence and the other probe. In addition, we could observe the higher S/B ratio with the higher concentration of a probe set (Figure $6 \mathrm{C}$ ). Use of higher probe concentration is desirable to assay larger interacting partners. The nearly straight shape of the kinetic curve observed might reflect the large dimension of Fn7-8, which would lower the reconstitution efficiency and subsequent luminescent reaction rate.

Collectively, considering the demerits and the merits, Fluc PCA using purified elements enables us a rapid and convenient detection of direct PPI with high confidence. 


\section{Conclusions}

The feasibility of Fluc PCA in vitro using purified elements was demonstrated for the first time. The assay was found to have high S/B ratio, and allow the detection of larger size of interacting partner than detectable by FRET. In spite of relatively weak thermostability, the assay will be applicable to handy detection of various PPIs in vitro including in vitro diagnostics and drug screening.

\section{Methods}

\section{Materials}

ATP and D-luciferin $\left(\mathrm{LH}_{2}\right)$ were from Sigma, St. Louis, MO. MOPS (3-(N-morpholino)propanesulfonic acid) was from Dojindo, Kumamoto, Japan. Rapamycin was from Wako Pure Chemical Industries, Osaka, Japan or LKT Laboratories, St. Paul, MN. Synthetic genes for E. coli codonoptimized human FKBP12, appended with Nco I / Sfi I and NotI sites at the 5' and 3' ends, respectively, were from Mr Gene GmbH, Regensburg, Germany. The plasmid pFH154 encoding human fibronectin cDNA was from Health Science Research Resources Bank (HSRRB), Osaka, Japan. Other reagents in the highest grade available were from Wako Pure Chemical Industries unless otherwise indicated.

\section{Construction of FKBP/FRB fused Fluc fragments}

The DNA fragment encoding Fluc was obtained by PCR using pGEX-Ppy vector [23] as a template, and primers LucNotG4SB (5'- gg cgc gcc GCG GCC GCC GGT GGT GGT GGT AGC ATG GAA GAC GCC AAA AAC ATA AAG-3') encoding a $\mathrm{G}_{4} \mathrm{~S}$ linker and NotI site (underlined) and LucXhoF (5'- g gcg cgc CTC GAG CTT TCC GCC CTT CTT GGC CT- $3^{\prime}$ ) containing XhoI site (underlined). Similarly, N- and C-terminal domain genes were amplified with primers LucNotG4SB and Luc437XhoF (5'-g gcg cgc CTC GAG GCG GTC AAC TAT GAA GAA GTG- $3^{\prime}$ ), and Luc394NotG4SB (5'- gg cgc gcc GCG GCC GCC GGT GGT GGT GGT AGC GGA CCT ATG ATT ATG TCC GG-3') and LucXhoF, respectively. The amplified fragments were cloned into pET32b (Merck, Darmstadt, Germany) between the NotI and XhoI sites, to give pET32/ Fluc, pET32/FlucN and pET32/FlucC, respectively. The synthetic genes encoding FKBP12 and FRB CDNAs were digested with $\mathrm{NcoI}$ and NotI, and the digested fragments inserted in pET32/FlucN and pET32/FlucC digested with the same enzymes each other, to give pET32/FKBP/FlucN, pET32/FKBP/FlucC, pET32/FRB/FlucN and pET32/FRB/ FlucC.

\section{Construction of FRB fused FlucN(1-398) fragments}

The DNA fragment encoding Fluc N-terminal domain (1-398) was obtained by PCR using pET32/FRB/FlucN as a template, and primers LucNotG4SB and Luc398XhoF $\left(5^{\prime}-\mathrm{t}\right.$ gtt tac ata CTC GAG cat aat cat agg tcc tct tac- $\left.3^{\prime}\right)$ containing $\mathrm{XhoI}$ site (underlined). The amplified fragments were cloned into $\mathrm{pET} 32 / \mathrm{FRB} / \mathrm{FlucN}$ between the NotI and XhoI sites, to give pET32/FRB/FlucN (1-398).

\section{Construction of $\mathrm{p} 53 / \mathrm{Mdm} 2$ fused Fluc fragments}

The DNA fragment encoding transactivation domain of p53 (residue 15-29) and two restriction sites (underlined) was obtained by thermal cycling using following oligonucleotides: p53NcoBack (5'-gg aat tCC ATG GCT AGT CAG GAA ACA TTT TCA GAC CTA TGG AAA C-3') and p53NotFor (5' $-\mathrm{g}$ gga ttc tGC GGC CGC GTT TTC AGG AAG TAG TTT CCA TAG GTC TG-3'). Mdm2 gene (residue 17-125) was obtained by PCR with human Mdm2 gene as a template and mdm2NocBack ( $5^{\prime}$-gg at tCC ATG GCT TCG GAA CAA GAG ACC C-3') and mdm2NotFor ( $5^{\prime}-\mathrm{g}$ gaa ttc tGC GGC CGC CTG CTG ATT GAC TAC TAC C-3') as primers. The amplified fragments were digested with $N c o I$ and NotI, and inserted to pET32/FKBP/FlucC and pET32/FRB/FlucN digested with the same enzymes, to give $\mathrm{pET} 32 / \mathrm{p} 53 / \mathrm{FlucN}, \mathrm{pET} 32 / \mathrm{p} 53 /$ FlucC, pET32/Mdm2/FlucN and pET32/Mdm2/FlucC.

\section{Insertion of $4 \times$ DDAKK between FKBP12 and FlucC} The two oligonucleotides DDAKK4_NotBack2 $\left(5^{\prime}\right.$-g gaa ttc GCG GCC GCA GAT GAT GCT AAA AAA GAT GCT AAA AAA GAT GAT GCC AAG AAG GAC GAC GC- $3^{\prime}$ ) containing NotI site (underlined), and DDAKK4 EagFor2 $\left(5^{\prime}-\mathrm{g}\right.$ gaa ttC GGC CGA TTT TTT AGC ATC ATC TTT TTT CGC GTC GTC CTT CTT GGC- 3') containing EagI site (underlined) were annealed and extended using a thermal cycler. The fragment was digested with EagI, and inserted into pET32/FKBP/FlucC digested with NotI, to give pET32/FKBP/4 × DDAKK/FlucC.

\section{Insertion of 7×DDAKK between FKBP12 and FlucC}

$\mathrm{pET} 32 / \mathrm{FKBP} / 4 \times \mathrm{DDAKK} /$ FlucC was amplified with the primers, DDAKK_VectorNotB (5'-GA CGA CGC CAA AAA AGA TGA TGC CAA GAA GG-3'), and DDAKK_ VectorNotF (5'-CT TTT TTA GCA TCA TCT GCG G-3'). The $4 \times$ DDAKK fragment inserted in $\mathrm{pET} 32 / \mathrm{FKBP} / 4 \times$ DDAKK/FlucC was amplified with the primers DDAKK LinkerB ( $5^{\prime}$-GA TGA TGC TAA AAA AGA TG- $\left.3^{\prime}\right)$ and DDAKK_LinkerF (5'-TT TTT TGG CGT CGT CTT TTT TCG CGT CGT C- $3^{\prime}$ ). The two amplified fragments were connected using In-Fusion HD cloning kit (Takara-Bio, Shiga, Japan), to obtain pET32/FKBP/8 $\times$ DDAKK/FlucC, which resulted in unexpected acquisition of $\mathrm{pET} 32 / \mathrm{FKBP} /$ $7 \times$ DDAKK/FlucC.

\section{Construction of FKBP/FRB fused with fluorescent proteins} The cDNAs for Ypet on pYpet-His (kindly provided by Dr. PS Daugherty) [24] and Cerulean [25] made from pEBFPN1 plasmid (Clontech, Takara-Bio) were amplified using 
specific primers with $5 /$-terminal NotI and $3{ }^{\prime}$-terminal XhoI sites, digested with $\mathrm{NotI}$ and $\mathrm{XhoI}$, and inserted into pET32/FKBP/FlucN and pET32/FRB/FlucN digested with the same enzymes, to give $\mathrm{pET} 32 / \mathrm{FKBP} /$ Cerulean and pET32/FRB/Ypet.

\section{Insertion of Fn7-8 as a rigid linker}

The sequence for Fn7-8 was amplified from human fibronectin cDNA with the primers Fn7EagBack $\left(5^{\prime}-\mathrm{g}\right.$ gaa $\mathrm{ttC}$ GGC CGC ACC ATT GTC TCC ACC AAC AAA C- $\left.3^{\prime}\right)$ and Fn8EagFor (5'-g gaa ttC GGC CGA TGT TTT CTG TCT TCC TCT AAG- $3^{\prime}$ ) each containing an EagI site. The amplified fragment was digested with EagI, and was inserted into pET32/FKBP/FlucC and pET32/FKBP/Cerulean digested with same enzyme, respectively.

\section{Expression and purification of probe proteins}

All the fusion proteins were expressed in E. coli BL21 (pLysS, DE3) (Novagen) as a thioredoxin and hexahistidinetagged protein. To purify the expressed fusion proteins, Talon metal affinity resin (Clontech) was used according to the manufacturer's instruction. Concentration of the purified protein was determined by CBB-stained SDS-PAGE co-loaded with various concentrations of BSA as a concentration standard. The protein added with final $15 \%$ glycerol was stored at $-80^{\circ} \mathrm{C}$ before use.

\section{Detection of PCA}

The purified probe proteins with or without rapamycin were suspended in $100 \mathrm{mM}$ MOPS, $10 \mathrm{mM} \mathrm{MgSO}_{4}$, $\mathrm{pH}$ 7.3. The mixture $(50 \mu \mathrm{l}$ each) was dispensed to a well of 96-well half well white plate (Corning-Costar, NY, USA). The light intensity was measured immediately after injection of $50 \mu \mathrm{l} 2 \times$ substrate solution $(40 \mathrm{mM}$ ATP and $150 \mu \mathrm{M} \mathrm{LH}_{2}$ in $100 \mathrm{mM}$ MOPS, $10 \mathrm{mM} \mathrm{MgSO}$, $\mathrm{pH}$ 7.3) with a periodical integration for $0.1 \mathrm{~s}$ using a luminometer Phelios AB-2350 (ATTO, Tokyo, Japan).

\section{FRET assay}

Fluorescence spectra were measured by F-2500 fluorescence spectrophotometer (Hitachi High-Technologies, Tokyo, Japan). Samples were diluted in $250 \mu \mathrm{l}$ PBS, pH 7.4. The mixture of $\mathrm{FKBP} /$ cerulean or $\mathrm{FKBP} / \mathrm{Fn} /$ cerulean and $\mathrm{FRB} /$ Ypet (40 $\mathrm{nM}$ each) was excited at $433 \mathrm{~nm}$, and the fluorescent spectra at $455-600 \mathrm{~nm}$ were recorded in the presence and absence of $40 \mathrm{nM}$ rapamycin.

\section{Additional files}

Additional file 1: Figure S1. Fluc PCA using a probe split at different site FRB/N (1-398). (A) SDS-PAGE of purified FRB/N (1-398). (B-E) Fluc PCA using FRB/N (1-398) and FKBP/C in 15, 50, 150, and 450 nM. (F) Signal/ background ratios of Fluc PCA using FRB/N (1-398) and FKBP/C.
Additional file 2: Figure S2. Examination of the probe stability. After incubating $100 \mathrm{nM}$ each of the probes with equimolar rapamycin (A) or without rapamycin (B) for 0 (lemon), 15 (yellow), 30 (orange) and 60 min (red) at $37^{\circ} \mathrm{C}, \mathrm{PCA}$ was performed as before. In (B), smaller scale of the vertical axis is used in the inset. (C) Comparison of the luminescent intensities with/without rapamycin after $60 \mathrm{~min}$ incubation.

\section{Abbreviations}

FKBP: FK506 binding protein; Fluc: firefly luciferase; Fn7-8: 7-8 domains of fibronectin type III; FRB: FKBP-rapamycin-associated protein;

FRET: fluorescence resonance energy transfer; GFP: green fluorescent protein; MOPS: 3-(N-morpholino)propanesulfonic acid; PCA: protein-fragment complementation assay; PPI: protein-protein interaction.

\section{Competing interests}

The authors declare that they have no competing interests.

\section{Authors' contributions}

YOM and $\mathrm{HU}$ designed and performed the research and wrote the manuscript. CIC performed the research and helped writing the manuscript. All authors read and approved the final manuscript.

\section{Acknowledgements}

We thank Keiichi Ayabe and Aoi Kimura for preparing Fluc-N and Fluc-C genes, and Yukiko Gotoh for human mdm2 cDNA. YOM was supported by SENTAN, Japan Science and Technology Agency, Japan.

\section{Author details}

${ }^{1}$ Department of Chemistry and Biotechnology, School of Engineering, The University of Tokyo, 7-3-1 Hongo, Bunkyo-ku, Tokyo 113-8656, Japan. ${ }^{2}$ The Japan Society for the Promotion of Science, 8 Ichiban-Cho, Chiyoda-ku, Tokyo 102-8472, Japan. ${ }^{3}$ Chemical Resources Laboratory, Tokyo Institute of Technology, R1-18, 4259 Nagatsuta-cho, Midori-ku, Yokohama 226-8501, Japan.

Received: 3 October 2012 Accepted: 15 March 2013

Published: 28 March 2013

\section{References}

1. Venkatesan K, Rual JF, Vazquez A, Stelzl U, Lemmens I, Hirozane-Kishikawa T, Hao T, Zenkner M, Xin X, Goh Kl, et al: An empirical framework for binary interactome mapping. Nat Methods 2009, 6(1):83-90.

2. Zhang QC, Petrey D, Deng L, Qiang L, Shi Y, Thu CA, Bisikirska B, Lefebvre C, Accili $D$, Hunter T, et al: Structure-based prediction of protein-protein interactions on a genome-wide scale. Nature 2012, 490(7421):556-560.

3. Shekhawat SS, Ghosh I: Split-protein systems: beyond binary proteinprotein interactions. Curr Opin Chem Biol 2011, 15(6):789-797.

4. Hu CD, Kerppola TK: Simultaneous visualization of multiple protein interactions in living cells using multicolor fluorescence complementation analysis. Nat Biotechnol 2003, 21(5):539-545.

5. Magliery TJ, Wilson CG, Pan W, Mishler D, Ghosh I, Hamilton AD, Regan L: Detecting protein-protein interactions with a green fluorescent protein fragment reassembly trap: scope and mechanism. J Am Chem Soc 2005, 127(1):146-157.

6. Luker KE, Smith MC, Luker GD, Gammon ST, Piwnica-Worms H, PiwnicaWorms D: Kinetics of regulated protein-protein interactions revealed with firefly luciferase complementation imaging in cells and living animals. Proc Natl Acad Sci U S A 2004, 101(33):12288-12293.

7. Paulmurugan R, Massoud TF, Huang J, Gambhir SS: Molecular imaging of drug-modulated protein-protein interactions in living subjects. Cancer Res 2004, 64(6):2113-2119.

8. Paulmurugan R, Umezawa Y, Gambhir SS: Noninvasive imaging of proteinprotein interactions in living subjects by using reporter protein complementation and reconstitution strategies. Proc Natl Acad Sci U S A 2002, 99(24):15608-15613.

9. Remy I, Michnick SW: A highly sensitive protein-protein interaction assay based on Gaussia luciferase. Nat Methods 2006, 3(12):977-979.

10. Kim SB, Ozawa T, Watanabe $S$, Umezawa Y: High-throughput sensing and noninvasive imaging of protein nuclear transport by using reconstitution of split Renilla luciferase. Proc Natl Acad Sci U S A 2004, 101(32):11542-11547. 
11. Porter JR, Stains $\mathrm{Cl}$, Jester BW, Ghosh I: A general and rapid cell-free approach for the interrogation of protein-protein, protein-DNA, and protein-RNA interactions and their antagonists utilizing split-protein reporters. J Am Chem Soc 2008, 130(20):6488-6497.

12. Chen J, Zheng XF, Brown EJ, Schreiber SL: Identification of an 11-kDa FKBP12-rapamycin-binding domain within the 289-kDa FKBP12rapamycin-associated protein and characterization of a critical serine residue. Proc Natl Acad Sci U S A 1995, 92(11):4947-4951.

13. Chiu MI, Katz H, Berlin V: RAPT1, a mammalian homolog of yeast Tor, interacts with the FKBP12/rapamycin complex. Proc Natl Acad Sci U S A 1994, 91(26):12574-12578.

14. Paulmurugan R, Gambhir SS: Combinatorial library screening for developing an improved split-firefly luciferase fragment-assisted complementation system for studying protein-protein interactions. Anal Chem 2007, 79(6):2346-2353.

15. Banaszynski LA, Liu CW, Wandless TJ: Characterization of the FKBP. rapamycin.FRB ternary complex. J Am Chem Soc 2005, 127(13):4715-4721.

16. Kussie PH, Gorina S, Marechal V, Elenbaas B, Moreau J, Levine AJ, Pavletich NP: Structure of the MDM2 oncoprotein bound to the p53 tumor suppressor transactivation domain. Science 1996, 274(5289):948-953.

17. Vassilev LT, Vu BT, Graves B, Carvajal D, Podlaski F, Filipovic Z, Kong N, Kammlott U, Lukacs C, Klein C, et al: In vivo activation of the p53 pathway by small-molecule antagonists of MDM2. Science 2004, 303:844-848.

18. Zhang J, Campbell RE, Ting AY, Tsien RY: Creating new fluorescent probes for cell biology. Nat Rev Mol Cell Biol 2002, 3(12):906-918.

19. Tsien RY: The green fluorescent protein. Annu Rev Biochem 1998, 67:509-544.

20. Wu P, Brand L: Resonance energy transfer: methods and applications. Anal Biochem 1994, 218(1):1-13.

21. Arai R, Ueda H, Kitayama A, Kamiya N, Nagamune T: Design of the linkers which effectively separate domains of a bifunctional fusion protein. Protein Eng 2001, 14(8):529-532.

22. Ohashi T, Galiacy SD, Briscoe G, Erickson HP: An experimental study of GFP-based FRET, with application to intrinsically unstructured proteins. Protein Sci 2007, 16(7):1429-1438.

23. Kitayama A, Yoshizaki H, Ohmiya Y, Ueda H, Nagamune T: Creation of a thermostable firefly luciferase with $\mathrm{pH}$-insensitive luminescent color. Photochem Photobiol 2003, 77(3):333-338.

24. Nguyen AW, Daugherty PS: Evolutionary optimization of fluorescent proteins for intracellular FRET. Nat Biotechnol 2005, 23(3):355-360.

25. Rizzo MA, Springer GH, Granada B, Piston DW: An improved cyan fluorescent protein variant useful for FRET. Nat Biotechnol 2004, 22(4):445-449.

doi:10.1186/1472-6750-13-31

Cite this article as: Ohmuro-Matsuyama et al: Demonstration of proteinfragment complementation assay using purified firefly luciferase fragments. BMC Biotechnology 2013 13:31.

\section{Submit your next manuscript to BioMed Central and take full advantage of:}

- Convenient online submission

- Thorough peer review

- No space constraints or color figure charges

- Immediate publication on acceptance

- Inclusion in PubMed, CAS, Scopus and Google Scholar

- Research which is freely available for redistribution 